\title{
Secure Software for Rail Circuits in the Form of Distributed Sensors
}

\author{
${ }^{1}$ Nikoloz Mgebrishvili, ${ }^{2}$ MaKsim IAVICH, ${ }^{3}$ GIORgI IASHVILI, ${ }^{4}$ AMIRAN NOdiA \\ ${ }^{1}$ Railway department, Georgian technical University, Tbilisi, GEORGIA \\ ${ }^{2}$ School of technology, Caucasus University, Tbilisi, GEORGIA \\ ${ }^{3}$ School of technology, Caucasus University, Tbilisi, GEORGIA \\ ${ }^{4}$ Railway department, Georgian technical University, Tbilisi, GEORGIA
}

\begin{abstract}
In order to improve the safety of train traffic, we propose to introduce into practice a new type of rail circuits, fiber-optic rail circuits. Fiber optic cable is very sensitive to external shocks, vibration and deformation, which can play a crucial role in the detection of mechanical damage of rails and wheel sets and also to improve the positioning of the rolling stock. The branches of the fiber optic cable not only serve as a conductor of information, but also serve as a sensor, as they can perceive vibration. An OTDR (Optical Time Domain Reflectometer) analyzes the backscattered light signal to determine the shape of the physical impact that caused the bending. From the time between the emission of the light signal and the receipt of the backscattered signal, the fault location is calculated. The authors offer the model of the system, which will check the security problems of the trains and the rail circuits. The software of the corresponding system is presented, using the simulation techniques. The authors present the pseudo code of the software. They also offer the testing environment for the software.
\end{abstract}

Keywords - fiber optic cable, light signal, rail circuits, sensor, signal processing, security.

Received: July 5, 2019. Revised: January 24, 2021. Accepted: February 5, 2021. Published: February 28, 2021

\section{Introduction}

Currently, large investments are being made in the development of international transport, which significantly increases the role of rail transport. Against the backdrop of growing traffic, we are faced with the need to improve traffic safety and monitoring system. The main problem here is to timely identify the problems of interaction between the rail and the wheelset, which in turn requires constant supervision.

The main task of railway engineering is to develop a monitoring system that can detect worn rails and wheelsets, determine the exact location of the damaged section of rails and damaged wheelsets, and track static and dynamic axial loads. Despite the fact that much work has been carried out in this direction and many devices have been created, the relevance of the research does not diminish.

For decades, the main means of managing railway infrastructure - railway sections and rails, to control their inoccupancy and serviceability have been electric track circuits, the conductors of which are the rails.

When using it, only the control of the vacancy of the block-sections takes place [1]. It generates information by determining such a simple fact as the presence or absence of a signal current on a railway track. Therefore it is functionally limited. In other words, it records the occupancy of the blocksection, but cannot provide information about the exact location of the rolling stock. Moreover, in order to detect damage to the rail, the rail must be broken so that there is no electrical contact between the fragments $[2,3]$. However, even performing these limited functions is technically quite difficult and expensive. Besides, their reliability is low and, ultimately, they are characterized by such serious drawbacks as unreliability and instability because of:
- Low ballast resistance;

- The need to turn on choke-transformers and other field devices to organize the reverse canalization.

- Traction current and locomotive signalization;

- The occurrence of a dangerous effect of traction current;

- Decentralized placement of devices;

- Limiting the information content of locomotive signaling, etc. [4].

Despite the use of modern digital and communication technologies, in many cases, modern electric rail circuits do not fully comply with the corresponding operating modes [5]. Emergency situations are especially aggravated in conditions of sharp weather changes and ballast pollution with electrically conductive impurities, changing of ballast resistance effects on the accuracy of the track circuit, which still remains a problem. If the rail chains are damaged, it is impossible to detect a crack (damage) or wear on the rails and wheelsets.

It is also difficult to control the rolling stock while driving. By solving this problem, it will be possible to protect the rails and axles of wheelsets from the effects of overloaded or unevenly loaded wagons and avoid possible emergency situations caused by them. This is especially important for railways with difficult terrain.

The most important step towards improving the train traffic safety and information management systems is the 
introduction of a new type of rail circuits - fiber-optic rail circuits $[6,7]$.

We can observe that over the past two decades [8], many attempts have been made towards improving existing control systems by introducing fiber optic cables.

As a result of long-term relationships with various research groups and manufacturers of fiber optic equipment we found out that the Turkish Railways has attempted to introduce fiber Bragg grating optical sensors (FBG sensors) to improve the reliability of monitoring in rail transport. As expected, the sensors showed very high sensitivity, but based on the results of this experiment, we came to a conclusion that made us refuse to use FBG sensors as the basis for a modern control system. The first reason here was the technology (FBG technology). FBG technology involves backscattering from each grating in the path of the light. Thus, the light intensity drops when passing through all FBG sensors. If we install several FBG sensors one after the other, most of them will be useless, since the light does not reach them, and the second reason is the reliability. Even with just a few FBG sensors, the picture was very difficult to analyze. A monitoring system requires a huge number of such sensors to operate, which in turn requires sophisticated software. All this reduces the reliability of the proposed monitoring system. [9].

These results prompted us to find ways to develop more effective ways to conduct monitoring.

\section{The Novel Approach}

Investigation of the features of a fiber optic cable showed that an optical cable is sensitive to external physical influences [10]. This is a very important property, since detection of thermal extension of rails, micro cracks and other mechanical damages requires high sensitivity. The sensitivity of an optical cable is manifested in the violation of the linearity of the light beam passing through it. Under physical impact, the cable deforms and vibrates. In this process we get the deviation of two categories, in the form of microbends and macrobends. Conducted light is scattered at the bend. Some of the scattered light returns to the light emitter. The OTDR (Optical Time Domain Reflectometer) device is built on this principle [11]. Actually, a fiber-optic cable turns into a sensor at a bend $[12,13]$. Based on the analysis of the backscattered signal, we determine the type of impact that caused the bending. The distance to the bend can be set with high precision based on the time required from sending a signal to receiving a scattered signal $[14,15]$.

The authors of the idea proposed to create a monitoring system using fiber optic cable.

If we establish a tight mechanical contact between the fiber optic cable and the rails, we will be able allows monitoring the entire rail.

With every physical impact on a cable segment, a bend occurs, and we can accurately assess the location of this impact. Thus, the fiber optic cable does the work of the sensor.

The same way we should detect damaged wheelsets, control the load on the axles of the rolling stock, detect overheated boxes in the rolling stock, and determine the place of rail wear and so on. In this case, we need to increase optic cable sensitivity to recognize different types of physical influences. Therefore, to increase contact area of rails and optic cable we have we need to make so-called "loop" with amplifier. Thus, we can locate these sensitive loops along the entire length. They will provide more diverse information, and by linking a specific damage to a specific waveform, we can precisely determine the damage type location.

The offered scheme is presented below. On the first and second rails, the sensors are located opposite each other. Let us discuss the system conditionally using the example of one rail.

Fig. 1 a block diagram of the system of the first rail: a single-core fiber-optic cable (1) is set along the rail and these branches make loops with mechanical amplifiers, so called, fiber-optic sensors $(2-1 \div 2$-n). The "loop" increases sensitivity of the cable to external influence.

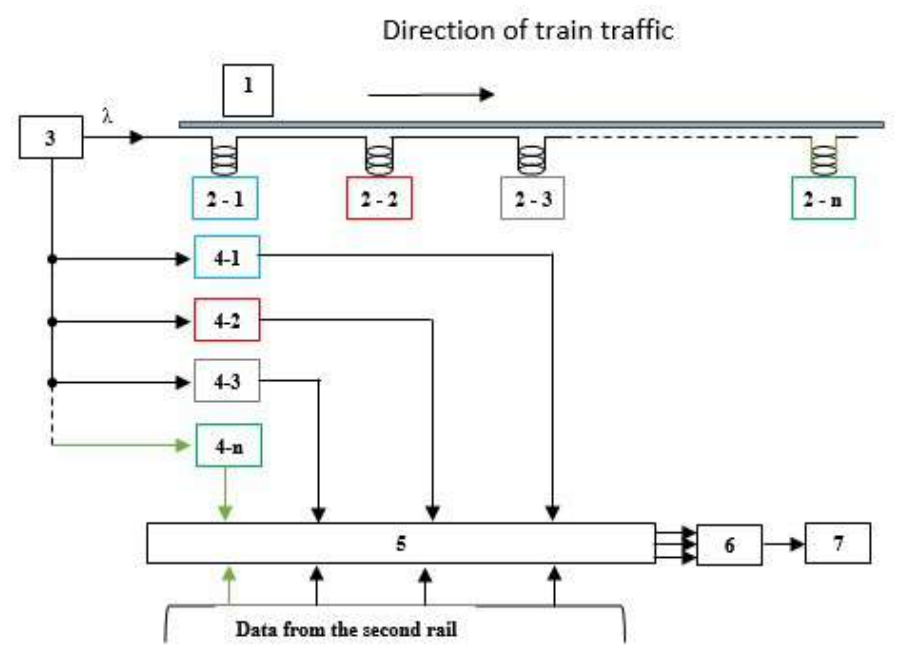

Fig. 1. System block-scheme.

In order to avoid simultaneous operation of two or more sensors, they are located at a well-defined distance from each other and from the reflectometers.

At the very beginning of the rail, there is an (3), $\lambda=1310$ $\mathrm{nm}$ or $\lambda=1550 \mathrm{~nm}$ wavelengths OTDR, connected to a chain of single-core fiber-optic cable tightly fixed along the rail. The OTDR generates an optical pulse and therefore determines their shape $\mathrm{S}$. It also calculates the exact coordinates of the pulse power drop (the distance from the OTDR to the point of the pulse power drop). 


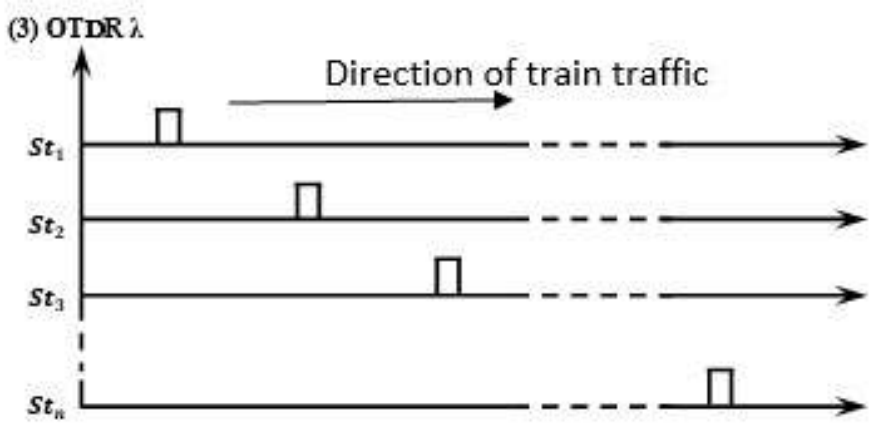

Fig.2 Register pulse triggering circuit scheme $(4-1 \div 4-n)$

OTDR (3) generates signals in the form of pulses Sn, When the wheels of the rolling stock impact on the sensor $(2-1 \div 2$ n). The ODDR output is connected to the registers $(4-1 \div 4-$ $\mathrm{n})$, each of which is connected to the corresponding sensor. Fig. 2: register pulse triggering circuit $(4-1 \div 4-n)$

Depending on the distance between the pulses of the reflectometer (3) $\left(\mathrm{t}_{\mathrm{n}}\right.$ interval), pulses $\left(\mathrm{St}_{1} \div \mathrm{St}_{\mathrm{n}}\right)$ are entered in the registers of the transmitter $(4-1 \div 4-n)$. Each register (4-1 $\div 4-n$ ) will work immediately after signals are sent to the register input.

These pulses are simultaneously transmitted to the computing unit (5) in the shape of ISn signals. Pulses in the form of IISn signals from the second rail are passed to the same computational unit, where the computation takes place:

1. After the wheelsets pass in the computing unit (5) in the direction of travel, the counters I4a $\div$ I 4 a 5 and II 4 a $1 \div$ II $4 \mathrm{a} 5$ count the number of pulses of the first five registers of both rails. after this, the number of counted pulses is spawned, for example I4a $\neq \mathrm{I} 4 \mathrm{a} 2=\mathrm{I} 4 \mathrm{a} 3=\mathrm{I} 4 \mathrm{a} 4=\mathrm{I} 4 \mathrm{a} 5=$ $\mathrm{II} 4 \mathrm{a} 1=\mathrm{II} 4 \mathrm{a} 2=\mathrm{II} 4 \mathrm{a} 3=\mathrm{II} 4 \mathrm{a} 4 \neq \mathrm{II} 4 \mathrm{a} 1$, the error is eliminated and the final result is issued. According to the developed algorithm, it determines the speed of movement; the number of rolling stock; type and number of cars in the train; the length of the rolling stock.

2. Checking and comparing analog signals, signal $\mathrm{S}$ with the standard $\Delta \mathrm{S}$ obtained from the corresponding register of the transmitter: from OTDRs(3) of the first and second guides, and the selection of distorted pulses and their following supply to the analysis unit (6);

3 . the received signals are processed in the analysis block according to the following logic:

a) If on a short section of the CD, the IS, IIS signals received from the sensors of the first and second rail become smaller at the same time in comparison with their standard in this section the rail is damaged;

b) If on a given section of the IC, IMS, the signals received from the first and second rail sensors are condensed together in comparison with their standard - heavy rolling stock was moving in this section;

c) If the first and second rail sensors $(2-1 \div 2-n)$ record similar changes in signals in comparison with the standard - there are carriages of different mass in the rolling stock;

d) In a certain area, the first and second rail sensors (2$1 \div 2$-n) detect various changes in I Sn and II Sn during the movement of a particular rolling stock, if $I \mathrm{Sn} \approx \Delta \mathrm{S}$ and II $\mathrm{Sn} \neq \Delta \mathrm{S}$, then the rolling stock is damaged the side where inequality is found;

4. According to a pre-formatted algorithm, the following are determined with high accuracy: rail load, worn rail, worn out rolling stock and wear quality;

Information enters the display (7) unit from the analysis unit (6).

\section{Algorithm and Pseudocode of the System}

In Fig. 3 and Fig. 4 shows the block diagrams of the operation algorithm of the computing unit (5) and the analysis unit (6).

Figure 3 shows the algorithm of the computing unit (5):

1. Input of signals ISn and IISn, From registers $(4-1 \div 4-n)$ of I and II rails

2. Calculation of signals coming from each register;

3. Error quantity comparison and elimination;

4. Formation of the number of wheel pairs;

5.Comparison of the analog signals $\mathrm{S}$ from the reflectometers I and II of rails (3) with the reference signal $\Delta \mathrm{S}$ and transmission of various (modulated) pulses to the analysis unit (6);

6. Train speed calculation between successive sensors at a known distance $\mathrm{x}$ and an interval $\tau$ when the wheelsets pass between these sensors;

7. Display of speed values;

8. Calculation of the number of received pulses a, taking into account the time intervals $\tau$ between them by the number of cars $\mathrm{N}=\mathrm{F}(\mathrm{a}, \tau)$ and determining the type;

9. Display of the number and type of carriages;

10. Summing up the lengths of the accepted number of cars and calculating the length of trains;

11. Display the trains lengths.

Figure 4 shows a description of the operation of the block diagram of the analysis unit algorithm (6):

1. Input of ISn and IISn values from the computing unit;

2. Detention of the damaged rail;

3. Definition of the CD segment and calculation of the degree of damage;

4. Train weight determination;

5. Calculation of the total mass of the train;

6. Delay of wagons of different weights;

7. Monitoring of the train by weight of wagons;

8., 9, Damaged wheelsets detention; 
10, 11. Calculation of the degree of damage to the wheelset;

12. Display of analysis results.

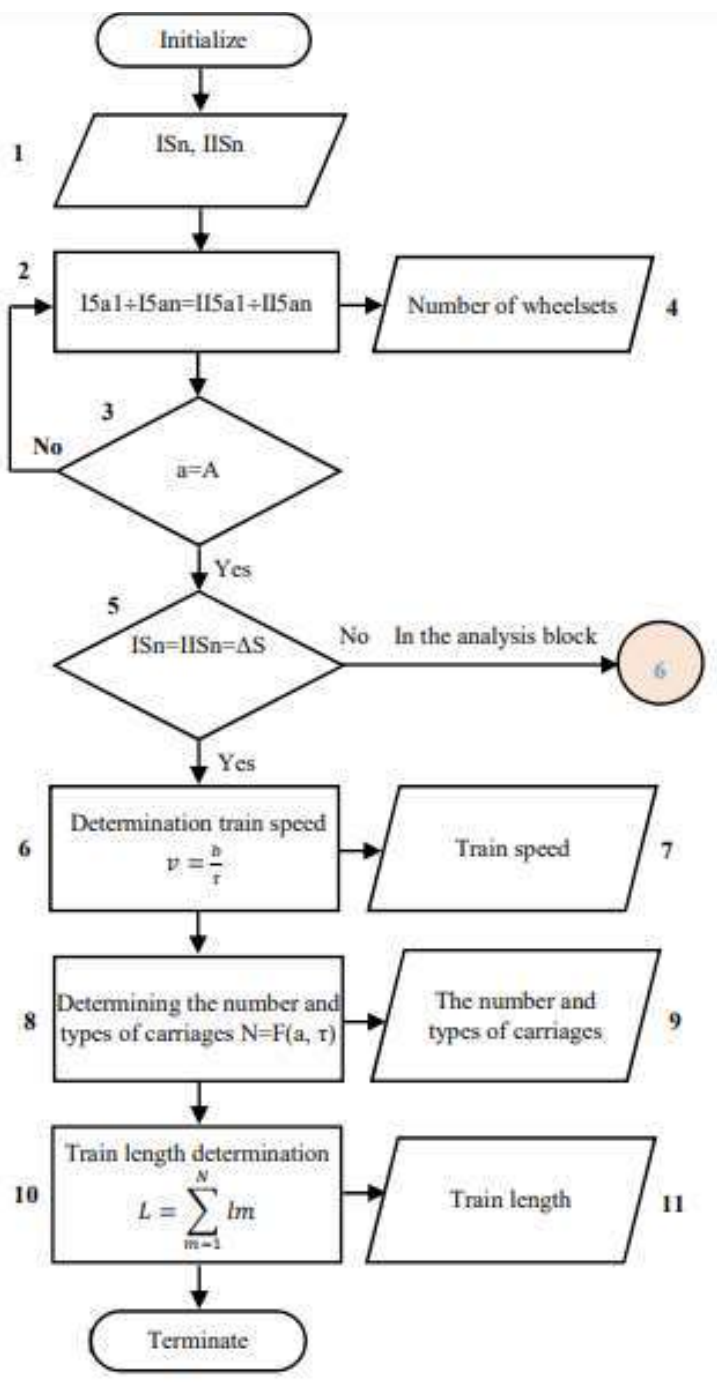

Fig. 3 The block scheme of the algorithm of the computing block (5)

The working algorithm of the analysis of the block 6 is presented on the figure 4 . In the algorithm is offered the definition of a damaged rail segment, the calculation of the total weight of the corresponding rolling stock and the identification of the corresponding rolling stock is performed.

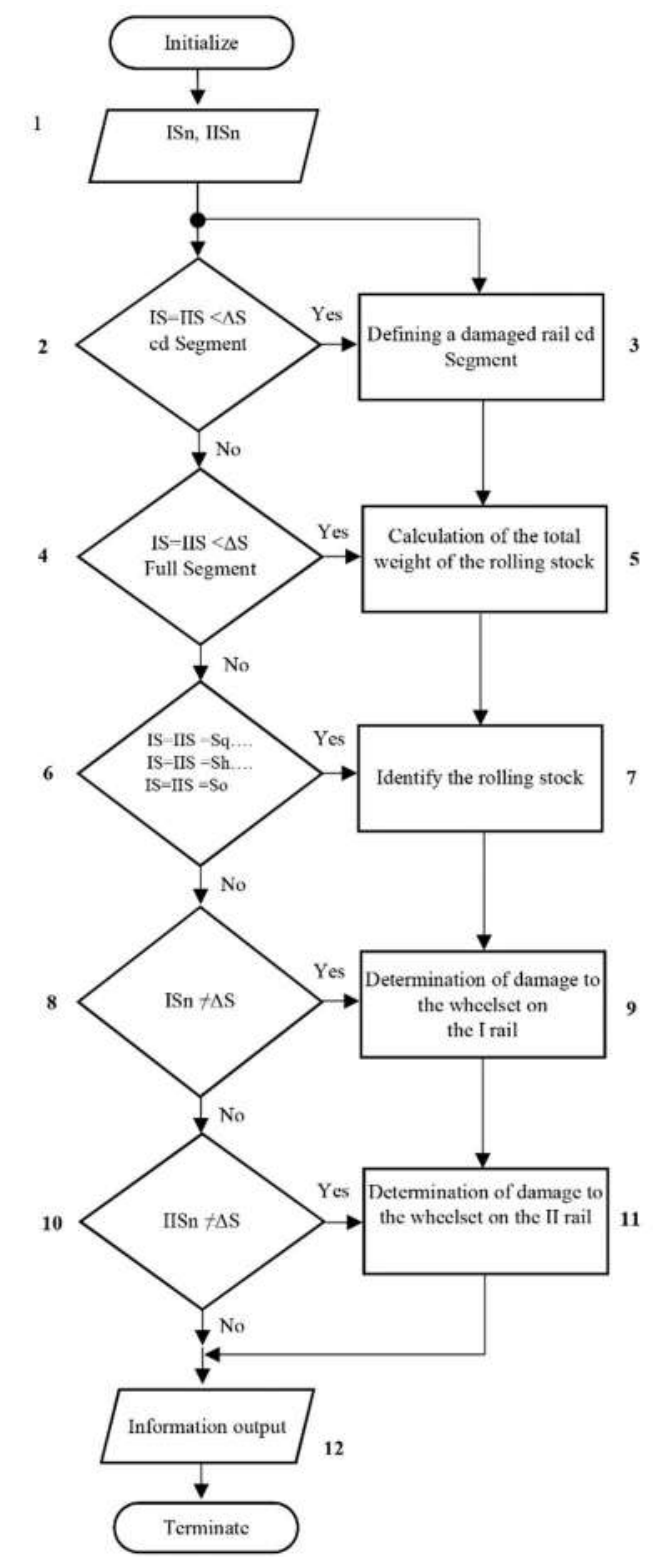

Fig. 4 The block diagram of the algorithm of the analysis unit (6)

The pseudocode of the system is offered below:

Importing the needed labs class Rail():

Def_init_(self):

Generating the number of wheels

Calculating the number of carriages

Making the log file of a spoiled rails

Making the log file of a spoiled wheels

Making the log file of the overweighed carriages

Creating list of sensors

Creating list of registers

Definition of the correct signal

Definition of deviation

Initialization of the overweighed carriages variable Initialization of the spoiled rales segment variable Initialization of the spoiled wheels variable def measure(self): 
\#Defining the method of analog signals measurement generating measurement

adding the measurements to the sensors list returning the list

def transfer(self):

\#Defining the method of passing information to registers passing the information to registers returning the registers list

def spoiled(self):

\#Defining the method of deviation identification identifying which wheels are the part of which carriage print the list of carriages checking if every wheel in the carriage has deviation:

identifying the index of each overweighed carriage

identifying the difference in the weight printing the list of overweighed carriage indexes and the difference in the weight adding the information to the $\log$

else:

removing the used wheels

checking which sequence of wheels have deviation: identifying the segment of wheels with deviation

identifying the indexes of the rail parts printing the list of the rail parts and the deviation

adding the information to the $\log$

else:

removing the used wheels

checking which wheel have deviation:

identifying the wheels with deviation

identifying the index of the wheels

printing the list of the wheels and the deviation

adding the information to the $\log$

closing the log file

class Interface:

adding the methods needed for the concrete interface

creating the object of the Rail class

running the spoiled method.

\section{Experiments}

In order to test the software the raspberry pi hardware were used. Raspberry hardware were used as the sensors, the experiments were done using 80 pieces of them.
The probability of the derivation was 0.1 , the 100 experiments were conducted. We present the results of the first 40 experiments.

The following results were received:

TABLE I.

\begin{tabular}{|c|c|c|c|}
\hline Experiment ID & $\begin{array}{c}\text { Overweighed } \\
\text { carriages }\end{array}$ & $\begin{array}{c}\text { Spoiled rail } \\
\text { segments }\end{array}$ & $\begin{array}{c}\text { Spoiled } \\
\text { wheels }\end{array}$ \\
\hline 1 & 0 & 25 & 50 \\
\hline 2 & 0 & 26 & 42 \\
\hline 3 & 0 & 21 & 44 \\
\hline 4 & 1 & 22 & 51 \\
\hline 5 & 0 & 11 & 26 \\
\hline 6 & 0 & 12 & 52 \\
\hline 7 & 1 & 29 & 40 \\
\hline 8 & 0 & 19 & 35 \\
\hline 9 & 0 & 32 & 30 \\
\hline 10 & 2 & 12 & 32 \\
\hline 11 & 1 & 14 & 41 \\
\hline 12 & 1 & 16 & 34 \\
\hline 13 & 3 & 18 & 54 \\
\hline 14 & 2 & 6 & 21 \\
\hline 15 & 1 & 12 & 14 \\
\hline 16 & 2 & 11 & 15 \\
\hline 17 & 0 & 5 & 44 \\
\hline 18 & 0 & 32 & 42 \\
\hline 19 & 0 & 16 & 32 \\
\hline 20 & 1 & 11 & 23 \\
\hline 21 & 0 & 13 & 43 \\
\hline 22 & 0 & 21 & 22 \\
\hline 23 & 1 & 22 & 23 \\
\hline 24 & 0 & 26 & 44 \\
\hline 25 & 1 & 28 & 47 \\
\hline 26 & 2 & 19 & 42 \\
\hline 27 & 0 & 11 & 22 \\
\hline 28 & 1 & 15 & 34 \\
\hline 29 & 0 & 21 & 55 \\
\hline 30 & 2 & 26 & 16 \\
\hline 31 & 3 & 22 & 22 \\
\hline 32 & 0 & 12 & 12 \\
\hline 33 & 0 & 22 & 18 \\
\hline 34 & 0 & 17 & 41 \\
\hline 35 & 0 & 6 & 32 \\
\hline 36 & 0 & 12 & 31 \\
\hline 37 & 0 & 8 & 29 \\
\hline 38 & 0 & 16 & 31 \\
\hline 39 & 1 & 18 & 32 \\
\hline 40 & 2 & 24 & 23 \\
\hline
\end{tabular}

\section{Conclusion}

Having studied similar patents, we have the opportunity to prove that the presented project has a great advantage - a universal system characterized by high measurement accuracy, extended functionality and a simplified structure compared to other similar methods.

Given schematic diagram of a multifunctional system based on fiber optic cable, which allows creating a new innovative method with which it will be possible to:

- detection of damaged wheelsets in rolling stock;

- detection of inter-permanent loading of rolling stock;

- Establishing the exact place of capture by the rolling stock of the inter-station block; rail;

- determination of the location of a worn out or damaged

- identification of rolling stock; 
- detection of overheated boxes in rolling stock;

- Rolling stock axles control.

The experiments show, that the scheme successfully works in the simulative environment.

Equipping these railways with presented systems will increase the efficiency of transportation and, consequently, budget revenues.

The developed scheme can also be successfully used for monitoring strategic facilities.

\section{Future Plans}

We are going to buy the needed hardware and to begin to test the system on Georgian railway. Our team is also working on integrating the machine learning into our algorithm. The idea is to collect the big amount of data by means of the experiments and to train the system.

\section{Acknowledgment}

This work was supported by Shota Rustaveli National Science Foundation of Georgia (SRNSFG) [grant number FR$18-4002]^{\prime \prime}$.

\section{References}

[1] Y.I.Polevoy - Basics of Railway Automatics and Telemechanics, Samara 2006, p.63-66.;

[2] Mgebrishvili N., Tatanashvili M., NadiradzeT., KekeliaK. - Increase of Railway Transportation Safety by a new method of Determination of Wheel Pair and Rail Wear and Damage (Eng) - 2007 ASME, RTDF 2007-46024 Technical Conference and Bearing Research Symposium - Chicago, Il, USA, 11-12 September, 2007; www.asmeconferences.org/RTDF2007/;

[3] N. Mgebrishvili, I. Garishvili, A. Dundua, K. Kutateladze, N. Kutubidze, G. Mghebrishvili - New Method of Determination of Wheel Pair's and Rail's Damage - Proceedings of Mechanics The International Scientific Conference, pp. 187-197, Tbilisi, 2016;

[4] Babaev M.M. , Grebenyuk V.Y - Analysis of The Influence of Electromagnetic Factors on The operation of Track Circuits., Collection of scientific papers of the Donetsk Institute of Railway Transport №28, 2011, p.75-82;

[5] Yu.N. Kulchin, V.A. Kolchinsky, O.T. Kamenev, Yu.S. Petrov - Fiberoptic measuring network based on quasi-distributed amplitude sensors for recording deformation effects - "Quantum Electronics", 43, No. 2 pp. 103-106. 2013;

[6] A. G. Kirilenko, N. A. Pelmeneva - Electric rail circuits - studies. allowance - Publishing House of Far Eastern State Transport University, - 94 p. 2006;

[7] Glyuk Martin (DE), Myuller Matias (DE) -Rail measuring system Patent RU No 2672 772. 2006

[8] Fiber Optical Sensors for High-Speed Rail Applications -Final Report $\begin{array}{lllll}\text { for High-Speed } & \text { Rail IDEA }\end{array}$ http://www.nationalacademies.org/trb/idea 2005;

[9] D. V. Efanov, G. V. Osadchy and V. V. Khóroshev, "Testing of Optical Sensors in Measuring Systems on Railway Marshalling Yard," 2018 IEEE East-West Design \& Test Symposium (EWDTS), Kazan, 2018, pp. 1-6, doi: 10.1109/EWDTS.2018.8524798.

[10] T. Norman - Integrated Security Systems Design (Second Edition), Elsevier Academic Press 2014, p.327-334;

[11] R. Anderson, Larry Johnson, Florian G.Bell - Troubleshooting OpticalFiber Networks: Understanding and Using Your Optical Time-Domain Reflectometer (Second Edition), Duwayne, Elsevier Academic Press, 2004, p.59-72;

[12] Ugale S.P., Mishra V. - Optimization of fiber Bragg grating length for maximum reflectivity // IEEE Intern. Conf. on Communications and Signal Processing (ICCSP). Pp. 28-32, 2011;

[13] Morozov, O. G. Synthesis of Two-Frequency Symmetrical Radiation and Its Application in Fiber

[14] O.G. Morozov et al. - Optical Structures Monitoring," [Fiber Optic Sensors] / - InTech, Rijeka, pp. 137-164,2012;

[15] M. Jin, Y. He, C. Jiang and Y. Liu - Fireworks: Channel Estimation of Parallel Backscattered Signals, 2020 19th ACM/IEEE International Conference on Information Processing in Sensor Networks (IPSN), Sydney, Australia, 2020, pp. 85-96, doi: 10.1109/IPSN48710.2020.0044.

[16] Q. Bai, Q. Wang, D. Wang, Y. Wang, Y. Gao, H. Zhang, M. Zhang and B. Jin - Recent Advances in Brillouin Optical Time Domain Reflectometry - /journal/ Sensors No19, 1862. P. 45. 2019;

\section{Creative Commons Attribution License 4.0 (Attribution 4.0 International, CC BY 4.0)}

This article is published under the terms of the Creative Commons Attribution License 4.0 https://creativecommons.org/licenses/by/4.0/deed.en_US 